\title{
EDITORIAL
}

\section{New technologies for social inclusion of people with psychosocial disabilities in the era of COVID-19 and beyond}

\author{
Mauro G. Carta, ${ }^{1}$ (iD Antonio E. Nardi, ${ }^{2}$ (iD Dinesh Bhugra ${ }^{3}$ \\ ${ }^{1}$ Dipartimento di Scienze Mediche e Sanità Pubblica, Università degli Studi di Cagliari, Cagliari, Italy. ${ }^{2}$ Laboratório Pânico e Respiração, \\ Instituto de Psiquiatria (IPUB), Universidade Federal do Rio de Janeiro (UFRJ), Rio de Janeiro, RJ, Brazil. ${ }^{3}$ Institute of Psychiatry, Psychology \\ and Neuroscience, King's College London, London, United Kingdom.
}

The impact of social isolation on mental well-being is well known. Isolation and loneliness can affect individuals as well as those around them, and this is especially the case during the COVID-19 pandemic. Since the use of new technologies is increasing in the pandemic, it is worth looking at how this is likely to change future clinical practice.

Social isolation affects health and is a known determinant of premature mortality. ${ }^{1}$ Negative stress due to social isolation affects hormonal and immunological responses and could induce epigenetic alterations. ${ }^{2}$ On the other hand, improving social networks has been found to improve health. ${ }^{2}$

Poverty and other social determinants are associated with social isolation. A poor social network and a diagnosed psychiatric disorder are interlinked, and their co-occurrence combines their effects. ${ }^{3}$ Although, in theory, online social networks should be able to provide a degree of support, this does not appear to be the case. Paradoxically, using Facebook and other social networks has been linked with poor perceived health, especially mental health and increased BMI. ${ }^{4}$ Shaya et al. ${ }^{4}$ point out that "The negative associations of Facebook use were comparable to or greater in magnitude than the positive impact of offline interactions, which suggests a possible tradeoff between offline and online relationships."4 Although increased use of online social networks leads to poor mental health, certain psychiatric disorders, such as attention deficit hyperactivity disorder, social phobia, hostility, depression, and suicidal ideation, have been linked to problematic Internet use and excessive use of online social networks. ${ }^{5}$ These associations should be explored further to clarify the impact of social media on well-being and health.

There is little doubt that the amount of social support derived from social media is difficult to measure. The number of "friends," "likes" or "followers" does not equate to social support. Although social media contact is often difficult to measure, the emotional support received in

Correspondence: Mauro G. Carta, Dipartimento di Scienze Mediche e Sanità Pubblica, Università degli Studi di Cagliari, SS 554, 09042 Monserrato, Cagliari, Italy.

E-mail: mgcarta@tiscali.it

Submitted May 27 2020, accepted May 31 2020, Epub Aug 312020. face-to-face contact can be assessed. Comparing online and face-to-face contact is difficult, perhaps because individuals with a large circle of friends may be more likely to use social media more frequently, as well as enjoy better health. ${ }^{5}$

Research on the use of communication technologies in medicine, clinical psychology and psychiatry is growing, including increasing evidence about online interventions for psychiatric disorders such as depression, anxiety and posttraumatic stress disorders, autism, and addictions. ${ }^{6}$ Given that they have been found effective as add-on treatments for mental health disorders, smartphone apps, videoconferencing, online forums, text-messaging and e-mails are being increasingly used for assessment and therapy in tele-mental health/e-mental health programs during the pandemic. ${ }^{6}$

Although some interesting studies have been published on complex and integrated social inclusion treatments for young and old people with psychosocial disabilities, this body of knowledge has not yet entered the daily armamentarium of psychiatrists and mental health rehabilitation services. ${ }^{2}$ The reasons for this delay are many, varying from lack of expertise to ethics, confidentiality, and privacy issues to a lack of training in specific competencies. Thus, although it has been shown that Internet social networks can help reduce social isolation during the pandemic, challenges remain.

The COVID-19 pandemic has led a dramatic increase in social isolation due to quarantining and self-isolation protocols. This has had devastating consequences for everyone but, above all, for those who were already vulnerable, such as people with psychosocial disabilities. ${ }^{7}$ Inevitably, many mental health day centers have closed, "whereas in residential facilities, patients who usually are free to come and go [...] have had to be confined in the facilities [...] These changes have produced considerable stresses on people with severe mental disorders."7 Italy is one of the most burdened countries by COVID-19 and has also seen one of the best-known human rights

How to cite this article: Carta MG, Nardi AE, Bhugra D. New technologies for social inclusion of people with psychosocial disabilities in the era of COVID-19 and beyond. Braz J Psychiatry. 2021;43:231-232. http://dx.doi.org/10.1590/1516-4446-2020-1175 
movement in history regarding mental health. However, associations of mental health service users and family members have reported that people with psychosocial disabilities are being abandoned due to the lockdown and quarantine. Consequently, mental health professionals and patients worldwide have found themselves using a range of methods, such as telephone and Internet, to communicate. ${ }^{8}$ This shift has been assisted by guidelines created by ad hoc consensus conferences or public health care systems. Nevertheless, many professionals have had little previous professional experience with these methods, and for some intervention types, efficacy and effectiveness studies are lacking or inadequate. Professionals are having to learn hurriedly, as they go, and often with limited resources. Paradoxically, this experience has opened a new world for most professionals and users. A way of communicating that, under normal circumstances, would have been complementary has now become the main channel for giving and receiving support, and it can access people who were difficult to reach with normal tools.

One of the few certainties that will remain after this COVID-19 pandemic ends is that social media has become a permanent, and often preferential, form of social contact. We live in a world of uncertainties, with weak predictions about what awaits us in the coming years. The advent of a vaccine may well restore a version of the world we knew (leaving only the pandemic's devastating geo-political-economic consequences). However, new waves of viral disease will radically change the way we live and communicate. In our medical work and mental health practice, one of the few things that is certain is that our means of communication have changed. People have discovered how Internet-based communication technologies can help overcome social isolation and, thus, they will continue to use it. This means that a robust, ethically controlled field of experimentation about these methods must grow and develop. Hence, proper ethical guidelines must be developed by professionals from various mental health sectors, especially regarding confidentiality and privacy issues. Tele- and e-mental health training with clearly defined competencies is an important first step, and we encourage regulatory bodies and national organizations to collaborate in its development.

\section{Disclosure}

The authors report no conflicts of interest.

\section{References}

1 Berkman LF, Syme SL. Social networks, host resistance, and mortality: a nine-year follow-up study of Alameda county residents. Am J Epidemiol. 1979;109:186-204.

2 Aiello AE. Invited commentary: evolution of social networks, health, and the role of epidemiology. Am J Epidemiol. 2017;185:1089-92.

3 Leigh-Hunt N, Bagguley D, Bash K, Turner V, Turnbull S, Valtorta N, et al. An overview of systematic reviews on the public health consequences of social isolation and loneliness. Public Health. 2017;152: 157-71.

4 Shakya HB, Christakis NA. Association of Facebook use with compromised well-being: a longitudinal study. Am J Epidemiol. 2017;185: 203-11.

5 Guedes E, Sancassiani F, Carta MG, Campos C, Machado S, King $\mathrm{AL}$, et al. Internet addiction and excessive social networks use: what about Facebook? Clin Pract Epidemiol Ment Health. 2016;12:43-8.

6 Zhou X, Snoswell CL, Harding LE, Bambling M, Edirippulige S, Bai X, et al. The role of telehealth in reducing the mental health burden from COVID-19. Telemed J E Health. 2020;26:377-9.

7 de Girolamo G, Cerveri G, Clerici M, Monzani E, Spirogatti F, Starace $F$, et al. Mental health in the Coronavirus Disease 2019 emergencythe Italian response. JAMA Psychiatry. 2020 Apr 30. doi: http:// 10.1001/jamapsychiatry.2020.1276. Online ahead of print.

8 Ćosić K, Popović S, Šarlija M, Kesedžić I. Impact of human disasters and COVID-19 pandemic on mental health: potential of digital psychiatry. Psychiatr Danub. 2020;32:25-31. 